Progress in Nuclear Science and Technology

Volume 5 (2018) pp. 66-69

\title{
ARTICE
}

\section{Cesium liquid-liquid extraction by calix-crown ethers: solvent effect}

\author{
Marie Simonnet*, Yuji Miyazaki, Shinichi Suzuki and Tsuyoshi Yaita \\ Japan Atomic Energy Agency, 2-4 Shirakata, Tokai-mura, Naka-gun, Ibaraki-ken, 319-1195, Japan
}

\begin{abstract}
Separation of cesium from nuclear waste can greatly decrease its decay heat. Calix-crown ethers are commonly used for liquid-liquid cesium extraction. However, their low lipophilicity makes them nearly insoluble in solvents like dodecane. In this study, experiments were conducted in different solvents with the final aim to find a way to predict solvent efficiency for cesium extraction. An extraction mechanism is proposed, and by fitting the data, this model provides empirical constants which can be related to solvent parameters, thus giving a basis for comparison and prediction. We finally discuss the best solvents for cesium extraction when using calix-crown ethers based on this study.
\end{abstract}

Keywords: cesium; liquid-liquid extraction; calix-crown ether; DOC[4]C6; solvent

\section{Introduction}

In order to improve the design of deep geological repositories, it has been proposed to remove ${ }^{90} \mathrm{Sr}$ and ${ }^{137} \mathrm{Cs}$ from the high level radioactive liquid waste (HLLW), for they are the two main heat generators after minor actinides. ${ }^{137} \mathrm{Cs}$ separation is also important for Fukushima water decontamination. Because the chemical behavior of cesium is very similar to potassium and sodium, which are found in substantial quantities in water, specific extractants for cesium need to be used to obtain selective extraction. Literature has focused on calix-crown-ether because of their high selectivity ability which can be tuned by the number of benzenes in the calix ring, and the number of ethers in the crownether. For cesium, the best are the calix[4]arene-crown-6 compounds in the 1,3 conformation [1].

One of them, di-octyloxycalix[4]arene-crown-6 (DOC[4]C6) has been thoroughly studied. It was also tested on genuine high-level waste at ATALANTE facility (CEA Marcoule, France) in a dodecane-TBP mixture. Cesium final recovery was higher than $99.99 \%$ [1]. TBP was necessary owing to the insolubility of the extractant in dodecane, but its presence led to a decreased selectivity, requiring a higher number of extraction stages. Accordingly, it could be profitable to find a more suitable solvent to improve such a process. i Many research studies on calix-crowns have been conducted in nitrophenyl alkyl-ether, mainly hexyl (NPHE) and octyl (NPOE), because of their excellent solvation properties [1]. However, their densities make them unsuitable to be applied as a solvent in an industrial process. Rais et al. [2] studied cesium

*Corresponding author. Email: simonnet.marie@jaea.go.jp extraction by DOC[4]C6 in many different solvents in order to find a correlation between the extraction rate for cesium and solvent properties. They found that the maximum distribution ratio almost linearly increases with dielectric constant and with water content in the organic phase in alcoholic media. This relationship, however, does not hold for the other kind of solvents that were studied (several ketones, dichloroethane and lauronitrile). Raut et al. [4] studied DOC[4]C6 in 6 different solvents. Chloroform showed a very poor extraction ability, 1-octanol a medium ability, and MIBK and NPOE very good abilities. PTMS and nitrobenzene also showed very good results but the extraction rate $D_{C s}$ was higher at $0.1 \mathrm{M}$ than at $3 \mathrm{M}$ $\mathrm{HNO}_{3}$, which makes the back extraction very difficult. The authors thus proposed to use Alamine-336 for the stripping. However, this modifier slightly decreases the extraction efficiency. Sharma et al. [5] studied DOC[4]C6 in a dodecane - 30\% isodecylalcohol mixture, which was found to be a suitable solvent even though the extraction efficiency is rather low.

Moyer [1] reported that the modification of the ether ring by the addition of benzene improved the extraction efficiency. For the present work, a derivative of DOC[4]C6, di-octyloxycalix[4]arene-benzo-crown-6 (DOC[4]BC6), was synthetized. Two other studies were found on the use of DOC[4]BC6 in liquid-liquid extraction: Kumar et al. [6] studied its behavior in dodecane $+30 \%$ NPHE and in dodecane $+30 \%$ isodecylalcohol, with slightly better results for the latter. Sachleben et al. [7] found that DOC[4]BC6 shows a slightly lower $\mathrm{D}_{\mathrm{Cs}}$ than DOC[4]C6 when extracting cesium from water to dichloroethane, which should lead to a better stripping efficiency.

The aim of this present study is to determine an 
optimal medium for cesium liquid-liquid extraction from HLLW with calix-crown-ethers. On this basis, systematic extraction studies were conducted in several pure or mixture solvents with DOC[4]C6 or DOC[4]BC6 extractants.

\section{Materials and experimental protocol}

Calix-crown-ethers were synthetized and purified in the laboratory (Figure 1). All solvents were from TCI.

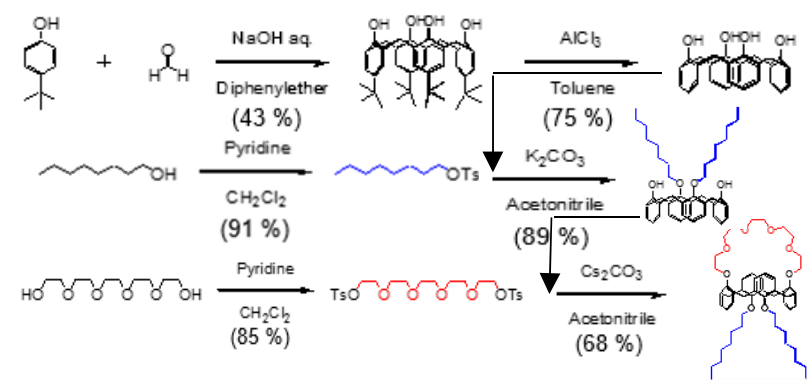

Figure 1. DOC[4]C6 synthesis route.

Equal volumes of each phase were mixed and shaken for 20 to 45 minutes at $25^{\circ} \mathrm{C}$ and $80 \mathrm{r} / \mathrm{m}$ with a $\mathrm{PC}$ Incubator Shaker model PC8-YS-8D. The samples were not pre-equilibrated to minimize nitric acid extraction. Samples were then centrifuged during 10 minutes at room temperature and analyzed by a Perkin Elmer ICPMS NexION 300D.

Results will be presented as distribution ratios $\mathrm{D}_{\mathrm{Cs}}$ :

$$
D_{C s}=\frac{[C s]_{t o t}-[C s]_{a q}}{[C s]_{\text {org }}}
$$

Throughout the paper, brackets stand for species concentrations, parenthesis for species activities, 'aq' and 'org' subscripts for respectively aqueous and organic phases, and 'tot' for total concentration.

\section{Results}

\subsection{Experimental data}

For every solvent, the variation of the distribution ratio with initial nitric acid concentration $\mathrm{C}_{\mathrm{HNO}}$ has been measured (Figure 2). The extractant used was DOC[4]BC6 $0.01 \mathrm{M}$. The solvents giving the highest distribution ratios were NPOE and 3methylcyclohexanone (MCHK) with values between 15 and 20 .

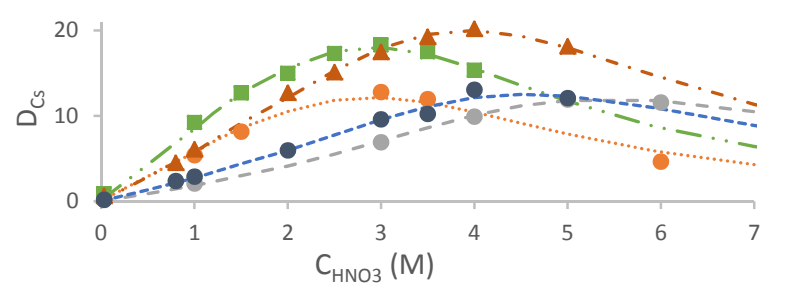

Figure 2. Cesium distribution ratio versus $\mathrm{C}_{\mathrm{HNO} 3}$ with DOC[4]BC6 0.01M in 1-octanol $+10 \%$ NPOE $\approx$, MCHK $\boldsymbol{\Delta}$ and n-octanol with $\mathrm{n}=1 \bullet, 2 \bullet$ and $3 \bullet$.
For each system, a maximum appears, which is obtained at different nitric acid concentrations $\left(\mathrm{C}_{\mathrm{HNO} 3} \mathrm{max}\right)$. Interestingly, the $\mathrm{C}_{\mathrm{HNO} 3}$ max of octanol is shifted towards higher nitric acid concentrations when the alcohol function is switched along the carbonated chain from carbon 1 to carbon 2 and carbon 3, while the maximum distribution ratio remains around 10 . However, increasing the solvent alkyl chain length decreases the distribution ratio, as can be seen in Figure 3 for linear ketones. The same behavior was found by Rais et al. [2] for linear alcohols. It is also noticeable that for both extractants, $\mathrm{C}_{\mathrm{HNO} 3}$ max shifts towards higher nitric acid concentrations while the maximum distribution value decreases.

When comparing the behavior of the two extractants in the same solvent (Figure 3), it can be seen that at low nitric acid concentrations, similar distribution ratios are obtained until around $3 \mathrm{M}$. They then show different values, because of their different $\mathrm{C}_{\mathrm{HNO}}$ max: DOC[4]C6 has its maximum around $4 \mathrm{M}$ while DOC[4]BC6 is around $5 \mathrm{M}$. This leads to higher distribution ratios for DOC[4]BC6 in all three solvents.

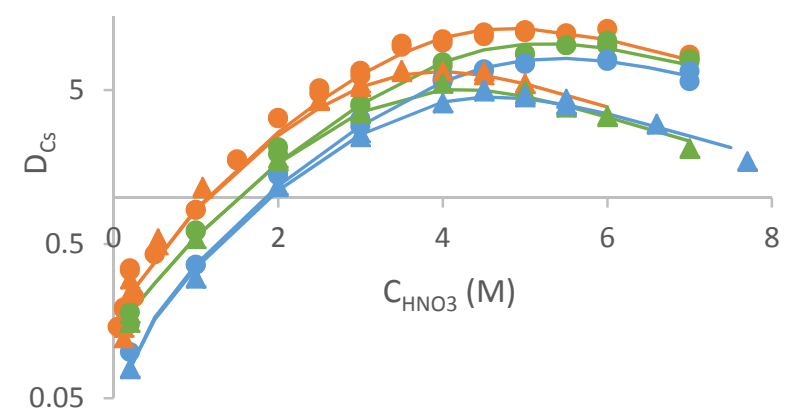

Figure 3. Cesium distribution ratio versus $\mathrm{C}_{\mathrm{HNO} 3}$ with DOC[4]BC6 - and DOC[4]C6 $\Delta \quad 0.01 \mathrm{M}$ in 2-nonanone (orange), 2-decanone (green) and 2-undecanone (blue).

The presence of this maximum distribution ratio is commonly found in previous literature for cesium extraction by calix-crown ethers. Several authors tried to qualitatively explain its presence [3-6], but no one provided a quantitative explanation. We thus decided to propose our own extraction model to understand the decrease of distribution ratios at high nitric acid concentrations.

\subsection{Modelling}

The extraction model which fits the data the most accurately consists of the competitive extraction of cesium and hydrogen ions as given by Eq. (2) and (3).

$$
\begin{gathered}
\mathrm{Cs}^{+}{ }_{\text {aq }}+\mathrm{NO}_{3}^{-}{ }_{\text {aq }}+\mathrm{L}_{\text {org }}=\left(\mathrm{CsLNO}_{3}\right)_{\text {org }} \\
\text { with } \mathrm{K}_{\mathrm{Cs}} \text { the equilibrium constant } \\
\mathrm{H}_{\text {aq }}^{+}+\mathrm{NO}_{3}^{-} \text {aq } \\
\text { with } \mathrm{K}_{\mathrm{HL}} \text { the equilibrium constant }
\end{gathered}
$$

The distribution ratio can then be calculated as a function of nitrate activity and extractant concentration (Eq. 4). Nitrate activities were calculated from the Davis and de Bruin dataset [8]. 


$$
D_{C s}=\frac{[C s]_{\text {org }}}{[C s]_{\text {aq }}}=\frac{K_{C s}\left(\mathrm{NO}_{3}^{-}\right)[L]_{t o t}}{1+K_{H L}\left(\mathrm{NO}_{3}\right)^{-}}
$$

Solvent effects are not considered directly in the mechanism, but are included in the equilibrium constants $\mathrm{K}_{\mathrm{Cs}}$ and $\mathrm{K}_{\mathrm{HL}}$, which represent formation in the aqueous phase and migration and stabilization in the organic phase of the two complexes. They are determined by fitting the data. For simplicity, the extracted compounds are written $\mathrm{CsLNO}_{3}$ and $\mathrm{HLNO}_{3}$.

The results of the analytical model are plotted in Figure 2 as dashed lines, showing very good agreement with the experimental data. To better check the validity of the model, data from literature was used, which also allowed us to increase the number of solvents to be compared. The analytical model could be applied on the experimental data for most of the solvents, except alkyl ketones for which the nitrates partial order of reaction was higher than 1. This result was found for both extractants and is likely to be caused by an interaction between the ketones and nitric acid. It is indeed an interesting property because the acidic condition difference between extraction and back-extraction is higher in these solvents. Another limitation of the analytical model is seen at low nitric acid concentration because it does not take into account the interactions with other species such as hydroxides, deprotonated solvents, and even water, which cannot be neglected.

The determination of $\mathrm{K}_{\mathrm{Cs}}$ and $\mathrm{K}_{\mathrm{HL}}$ for each possible solvent is an interesting way to compare and predict their efficiencies, that is to say the highest $\mathrm{D}_{\mathrm{Cs}}$ that can be reached. Best solvents have a high $\mathrm{K}_{\mathrm{Cs}}$ and a low $\mathrm{K}_{\mathrm{HL}}$.

\subsection{Solvent extraction efficiency prediction}

In order to predict the efficiency of a solvent, $\mathrm{K}_{\mathrm{Cs}}$ and $\mathrm{K}_{\mathrm{HL}}$ were plotted versus several solvent parameters, such as dielectric constant, water solubility in the solvent or Hansen parameters. Unfortunately, these parameters were not available in literature for all the studied solvents. Moreover, estimations had to be done for mixtures of solvents. For the dielectric constant, P. Wang and A. Anderko [9] equations for polarizability was used.
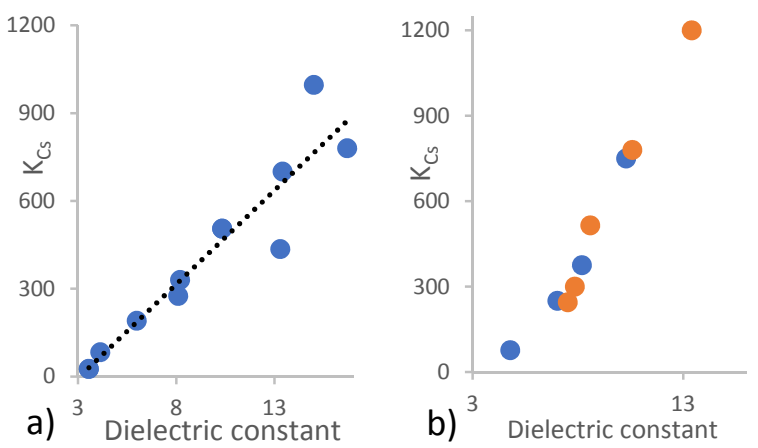

Figure 4. $\mathrm{K}_{\mathrm{Cs}}$ as a function of dielectric constant with a) DOC[4]C6, determined from own experiments and literature data [2-5] and b) DOC[4]BC6, determined from own experiments and Kumar et al. data [6], with orange dots being the calculated dielectric constant for mixture solvents and blue dots being pure solvents dielectric constant.
No correlation could be found with the Hansen parameters, showing there is no direct link between the extractant solubility and extractability. This is also proved by the comparison between chloroform and octanol, for which high solubility but low distribution ratios were obtained from chloroform and vice versa for octanol. An affine function was found between $\mathrm{K}_{\mathrm{Cs}}$ and the dielectric constant, as shown in Figure 4. This leads to two conclusions: polar solvents provide higher cesium extraction ability, and there is a minimum dielectric constant below which no extraction can be achieved.

We expected $\mathrm{K}_{\mathrm{HL}}$ to be somewhat correlated with water solubility in the solvent, as it represents nitric acid extraction. However, while aliphatic alcoholic solvents do show a linear trend with water content in the solvent, other solvents such as dichloroethane or cyclic solvents show no such trend. Measurementof the extracted amount of nitric acid by the diluents were performed, but once again no correlation was found. Another possibility is that this quantity is related to nitrates distance to cesium, which varies with diluent nature. A linear correlation can also be found between $\mathrm{K}_{\mathrm{Cs}}$ and $\mathrm{K}_{\mathrm{HL}}$ for aliphatic alcoholic solvents, which indicates that cesium and hydrogen ions are extracted through the same mechanism in these solvents (Figure 5). Interestingly, extraction in cyclic solvents shows an increase of $\mathrm{K}_{\mathrm{Cs}}$ relative to $\mathrm{K}_{\mathrm{HL}}$ as compared to aliphatic ones, which means there is an additional effect improving cesium extraction over proton extraction. Consequently, cyclic solvents are better than aliphatic solvents for cesium extraction.

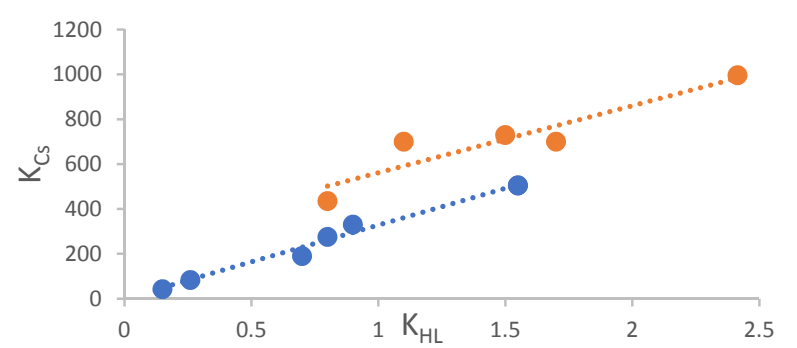

Figure 5. $\mathrm{K}_{\mathrm{Cs}}$ versus $\mathrm{K}_{\mathrm{HL}}$ in DOC[4]C6 for aliphatic alcoholic solvents $\bullet$ and cyclic solvents $\bullet$.

\subsection{Choice of solvent}

We have shown in the previous section that polar cyclic solvents yield higher cesium extraction. However, solvent hydrodynamic parameters need to be considered before they can be used for industrial purposes, especially in the nuclear industry where safety requirements are very high. Some of these parameters are listed in Table 1.

The cyclic solvents that have been studied by Rais et al. [2], whose results were used in the previous calculations, have low flash points so they cannot be of practical use. NPOE has a density too close to water to be used directly as a solvent, and its mixture with chloroform gives a low distribution ratio. Good results were obtained when mixed with 1-octanol, however NPOE and 1-octanol are respectively denser and less 
Table 1. List of solvents used in this study, data from ChemSpider.com; solubility is given as a qualitative description only. TBP and 2-NPOE were used as modifiers in respectively 70\% dodecane or 70\% 1-octanol, and $80 \%$ chloroform or $90 \% 1-$ octanol. Precipitate formed in TBP/dodecane so no results could be obtained. Both results are given for 2-NPOE.

\begin{tabular}{|c|c|c|c|c|c|c|c|c|}
\hline solvents & $\begin{array}{c}\text { boiling } \\
\left.\text { point } \mathbf{(}^{\mathbf{0}} \mathbf{C}\right)\end{array}$ & $\begin{array}{c}\text { melting } \\
\left.\text { point } \mathbf{(}^{\mathbf{0}} \mathbf{C}\right)\end{array}$ & $\begin{array}{c}\text { flash } \\
\left.\text { point } \mathbf{(}^{\mathbf{0}} \mathbf{C}\right)\end{array}$ & density & $\begin{array}{c}\text { solubility } \\
\text { in water }\end{array}$ & $\begin{array}{c}\text { solubility of } \\
\text { extractant }\end{array}$ & \multicolumn{2}{|c|}{ DOC[4]BC6 0.01M } \\
\hline 1-octanol & 195 & -16 & 81 & 0.824 & $0.54 \mathrm{~g} / \mathrm{L}$ & low & 12.6 & $\mathrm{D}_{\mathrm{Cs}} \max$ \\
\hline 2-octanol & 179 & -38 & 76 & 0.821 & $1.12 \mathrm{~g} / \mathrm{L}$ & low & 12.2 & 4.5 \\
\hline chloroform & 60 & -63 & - & 1.489 & $2.1 \mathrm{~g} / \mathrm{L}$ & high & 3 & 4.5 \\
\hline dodecane & 216 & -10 & 74 & 0.75 & $0.11 \mathrm{mg} / \mathrm{L}$ & very low & - & - \\
\hline 2-NPOE & 197 & - & 110 & 1.036 & $0.27 \mathrm{mg} / \mathrm{L}$ & very high & $4 / 17.5$ & 3 \\
\hline TBP & 289 & -80 & 146 & 0.973 & $7.4 \mathrm{mg} / \mathrm{L}$ & high & 10 & 5 \\
\hline 2-nonanone & 195 & -15 & 64 & 0.826 & $0.176 \mathrm{~g} / \mathrm{L}$ & very high & 12.2 & 5 \\
\hline 2-decanone & 210 & 3 & 86 & 0.824 & $46.4 \mathrm{mg} / \mathrm{L}$ & very high & 9.7 & 5.5 \\
\hline 2-undecanone & 231 & 13 & 89 & 0.825 & $19.7 \mathrm{mg} / \mathrm{L}$ & very high & 8.1 & 5.5 \\
\hline 3-MCHK & 169 & -73.5 & 48 & 0.9 & $5.1 \mathrm{~g} / \mathrm{L}$ & very high & 20.2 & 4 \\
\hline
\end{tabular}

dense than water, so it can lead to third phase formation. Octanol gives satisfactory results, though extractant solubility is rather low and solubility in water slightly high. Because the back-extraction behavior is not so good, Alexova et al. proposed to use di-octyloctanamide or di-hexyloctanamide as a stripping agent [3]. This study also showed that the maximum distribution ratio can be shifted towards higher nitric acid concentrations by moving the alcoholic function, thus providing lower distribution ratios at higher $\mathrm{pH}$. The most balanced solvents that were studied are alkylketones, because they have reasonably high flash points, high solubilization of extractant but low solubility in water, and provide satisfactory distribution ratios. The apparent order of the nitrates higher than one previously mentioned also provides better back-extraction behavior than other solvents.

\section{Conclusion}

A series of cesium extraction experiments were conducted in several solvents with two different extractants, DOC[4]C6 and DOC[4]BC6. An extraction mechanism was proposed which consists of the competitive extraction of cesium and hydrogen ions. The analytical model fitted the data accurately when using two empirical constants, $\mathrm{K}_{\mathrm{Cs}}$ and $\mathrm{K}_{\mathrm{HL}}$, whose values depend on both solvent and extractant natures. These constants can be related to solvent parameters such as dielectric constant. The final results showed that polar cyclic solvents yield the highest distribution ratios. However, their bad hydrodynamic behavior makes them unsuitable for industrial use, thus the best choice was determined to be alkyl-ketones because their properties suit the safety requirements of the nuclear industry. To be able to be used in industry, studies need to be conducted on their resistance towards hydrolysis and radiolysis.

\section{References}

[1] J.-F. Dozol and R. Ludwig, Ion exchange and solvent extraction: a series of advances vol. 19, chapter 4 Extraction of Radioactive Elements by
Calixarenes, B.A. Moyer, Taylor \& Francis Group, (2010), pp.195-318, ISBN 978-1-4200-5969-4.

[2] J. Rais, S. Tachimori, E. Yoo, J. Alexova and M. Bubenikova, Extraction of radioactive $\mathrm{Cs}$ and $\mathrm{Sr}$ from nitric acid solutions with 25,27-bis(1octyloxy)calix[4]-26,28-crown-6 and dicyclohexyl18-crown-6: effect of nature of the organic solvent Separ. Sci. Technol. 50 (2015), pp.1202-1212.

[3] J. Alexova, M. Sirova, J. Rais, S. Suzuki, M. Hirata, T. Kimura and S. Tachimori, Development of a process for the extraction of ${ }^{137} \mathrm{Cs}$ from acidic HLLW based on crowncalix extractant - use of dialylamide modifier, Proceedings of ISEC 2008, Tucson (2008).

[4] D. Raut, P. Mohapatra, M. Choudhary and S. Nayak, Evaluation of two calix-crown-6 ligands for the recovery of radio cesium from nuclear waste solutions: solvent extraction and liquid membrane studies, J. Membrane Sci. 429 (2013), pp.197-205.

[5] J. Sharma, A. Kumar, V. Kumar, S. Pahan, C. Janardanan, V. Tessi and P. Wattal, Process development for separation of cesium from acidic nuclear waste solution using 1,3dioctyloxycalix[4]arene-crown-6 + isodecyl alcohol/n-dodecane solvent, Sep. Purif. Technol. 135 (2014), pp.176-182.

[6] V. Kumar, J. Sharma, P. Achuthan and R. Hubli, Selective separation of cesium from simulated high level liquid waste solution using 1,3-dioctyloxy calix[4]arene-benzo-crown-6 J. Radioanal. Nucl. Ch. 299 (2014), pp.1547-1553.

[7] R.A. Sachleben, J.C. Bryan, L.N. Engle, J.T. Haverlock, P.B. Hay, A. Urvoas and B.A. Moyer, Rational design of cesium-selective ionophores: dihydrocalix[4]arene crown-6, ethers, Eur. J. Org. Chem. (2003), pp.4862-4869.

[8] W.J. Davis and H.J. de Bruin, New activity coefficients of 0-100 percent aqueous nitric acid, $J$. Inorg. Nucl. Chem. 26 (1964), pp.1069-1083.

[9] P. Wang and A. Anderko, Computation of dielectric constants of solvent mixtures and electrolyte solutions, Fluid Phase Equilibr. 186 (2001), pp.103-122. 